\title{
Philosophical Implications of Inflationary Cosmology
}

\author{
Joshua Knobe, Ken D. Olum, and Alexander Vilenkin
}

\begin{abstract}
Recent developments in cosmology indicate that every history having a nonzero probability is realized in infinitely many distinct regions of spacetime. Thus, it appears that the universe contains infinitely many civilizations exactly like our own, as well as infinitely many civilizations that differ from our own in any way permitted by physical laws. We explore the implications of this conclusion for ethical theory and for the doomsday argument. In the infinite universe, we find that the doomsday argument applies only to effects which change the average lifetime of all civilizations, and not those which affect our civilization alone.
\end{abstract}

\section{Contents}

1 Introduction 2

2 Physics Backoround 3

2.1 The number of possible histories is finite . . . . . . . . 3

2.2 The universe is infinite . . . . . . . . . . . . 4

2.3 Everv possible historv occurs an infinite number of times 5

$\begin{array}{lll}3 & \text { Frequency and Probability } & 6\end{array}$

4 Inflation Contrasted $\quad 8$

4.1 Modal realism . . . . . . . . . . . . . . . . 8

4.2 Actualism . . . . . . . . . . . . . . . 10

4.3 Eternal recurrence . . . . . . . . . . . . . . . . 11

5 Ethical Implications 13

6 Universal Doomsdav 16

6.1 Application to our civilization in particular . . . . . . 18

6.2 Universal vs. particular dooms . . . . . . . . . . . 20

6.3 Practical applications . . . . . . . . . . . 20

$\begin{array}{lll}7 \text { Concluding Remarks } & 21\end{array}$ 


\section{Introduction}

It is said that the ancient Greek philosopher Diodorus Cronos once put forth a powerful argument for a peculiar view about the relationship between the possible and the actual. Diodorus claimed that everything that could possibly happen is either occurring right now or will occur at some point in the future. His claim, in other words, was that there are no unrealized possibilities. Unfortunately, the works of Diodorus have been lost, and although a number of modern philosophers have tried valiantly to reconstruct his argument, no one really knows exactly how it was supposed to go.

Nonetheless, we think that Diodorus's conclusion was essentially correct, and we will here provide a new, entirely modern argument for it. Unlike the original argument of Diodorus, however, our argument draws on inflationary cosmology and quantum mechanics. It follows from inflationary cosmology that the universe is infinite and can therefore be divided into an infinite number of regions of any given size. But it follows from quantum theory that the total number of histories that can occur in any one of these regions in a finite time is finite. We draw on these two premises to argue for our central conclusion: that all possible histories are realized in some region of the universe.

This conclusion must be understood in a special sense. First, note that we are reserving the word 'possible' for physical possibilities. Thus, although it might be metaphysically possible for a system to violate strict physical laws, such a system would not count as 'possible' in our sense. Second, we do not mean to say that anything that can possibly happen to a token individual will actually happen to that individual. The conclusion is rather that anything that can happen to a particular type of individual will actually happen to some token of that type. So, for example, we do not claim that everything that can possibly happen to you will actually happen to you. The claim is only that everything that can possibly happen to you will actually happen to some qualitative duplicate of you.

Ultimately, our argument is more a scientific theory than a philosophical account, and it has already been presented as such elsewhere (Garriga \& Vilenkin [2001]). Still, we feel that the theory has important implications for issues that have traditionally been the concern of philosophers. This paper, written jointly by two physicists and a philosopher, explores these philosophical implications.

We proceed in two steps. First, we provide a condensed, non-technical 
explanation of the argument. Then we explore the implications of this argument for questions about modality, ethics, and doomsday.

\section{Physics Background}

The assertions that the universe is infinite and that the number of possible histories in a finite spacetime region is finite are crucial for our argument. Here, we shall briefly discuss the physical origin of these claims and provide some references where further details can be found.

\subsection{The number of possible histories is finite}

Suppose we pick a region of space and an interval of time. This defines a region of spacetime. We want to consider histories that can occur in this spacetime region. If we divide the space in such a region into small subregions, we can define a history as a specification of the contents of each subregion at successive moments of time.

Quantum mechanics assigns a probability to each of the histories, and we say that a history is possible if its probability is not equal to zero. This includes a very wide class of histories, since in quantum mechanics anything that is not strictly forbidden has a nonzero probability. The only histories that are excluded are the ones that violate some exact conservation laws, like the conservation of energy or of electric charge.

It can be shown, however, that there are only finitely many distinct histories that can occur in any finite spacetime region. One might think that the subregions and the intervals between moments of time could be made arbitrarily small, and the contents specified arbitrarily precisely, so the number of possibilities should be infinite, and of course in classical mechanics that would indeed be so. But in the quantum mechanical world the situation is different.

In quantum mechanics, if two histories are too similar, there is the possibility of interference between them. In that case, it is not meaningful to say that the two histories are alternative possibilities. Instead, both possibilities together contribute to the outcome.

The concept in quantum mechanics that corresponds to the ordinary idea of alternative possibilities is that of decoherent histories (Gell-Mann and Hartle, [1993]). If the subregions and their values are specified suffi- 
ciently coarsely, the resulting histories will decohere, meaning that they do not interfere with each other and can be meaningfully interpreted as classical alternatives. When we discuss the set of possible histories, we mean the set of decoherent histories, which are mutually exclusive possibilities for the evolution of a region. In order to have decoherence between the histories, the subregions cannot be too small and their values cannot be too finely specified, and as a result the number of decoherent histories is finite. For more details see Garriga and Vilenkin ([2001]).

Suppose, for example, that we have a box containing a radioactive atom, which decays by emitting an alpha particle. We observe the box over a finite time T. Histories of the box can be defined by dividing it into little cells and subdividing $\mathrm{T}$ into small intervals. If the decay occurs during the time $\mathrm{T}$, then at some point an alpha-particle will appear in one of the cells, and then move on to other cells, as it propagates away from the atom. A possible history is given by specifying the cells containing the atom and the alphaparticle at each time interval. To assure decoherence, the cell sizes need to be chosen greater than the de Broglie wavelength of the alpha-particle; there is a similar lower bound on the time interval.

\subsection{The universe is infinite}

The claim that the universe is infinite is a consequence of the theory of inflation. This theory began as a speculative hypothesis when it was proposed by Alan Guth ([1981]), but it is now well on its way to becoming one of the cornerstones of modern cosmology. The central role in the theory is played by a peculiar form of matter-known as 'false vacuum'-which is characterized by high energy and strong repulsive gravitational field. Here, the word 'false' alludes to the fact that this type of vacuum is unstable and decays into ordinary (true) vacuum. Inflation is an epoch of super-fast, accelerated cosmic expansion, driven by the repulsive gravity of false vacuum. Decay of the false vacuum marks the end of inflation and plays the role of the big bang in this theory.

One of the striking aspects of inflation is that, generically, it never ends in the entire universe. False vacuum decay is a probabilistic process; it does not occur everywhere simultaneously. In practically all models of inflation, false vacuum regions grow due to expansion faster than they decay. This means that the total volume of such regions in the universe keeps growing without bound. Thus, inflation is a runaway process, which stopped in our 
region, but still continues in other parts of the universe (Vilenkin [1983]; Linde [1986]; for a recent review, see Guth [2000]).

Post-inflationary regions like ours form 'island universes' in the inflating sea. As seen by an observer in the false vacuum region, the false vacuum is always decaying and so each island universe becomes larger and larger. Different parts of the universe spend different amounts of time in the inflating state.

However, from the point of view of an observer like us in an island universe, the Big Bang is the time at which inflation ended, and our notion of time is the time since then. Our island universe is infinite, in the sense that the volume of space where the time since the big bang is the same as that time here goes on forever and so is infinitely large. One cannot travel from an island universe to the inflating sea, because that would require going backward in time, nor can one travel to another island universe.

The eternally inflating spacetime contains an infinite number of island universes. However, since each island universe is itself spatially infinite, it is sufficient for our purposes to consider a single island universe.

Remarkably, the entire universe, which contains all these infinite island universes, may be finite. The apparent contradiction is resolved due to the fact that the internal notion of time in island universes is different from the 'global' time that one has to use to describe the entire spacetime. The volume of the universe at a particular global time may be finite, but the volume in an island universe at the time of the Big Bang in that universe (or any later time) is infinite.

When we discuss the history of a region of our island universe we include only the history since the Big Bang, i.e., since inflation ended. We do not include the time that the region spent in the false vacuum. That time has no effect on later events, since the state of a region at the end of inflation has no dependence on the amount of time it spent inflating.

\subsection{Every possible history occurs an infinite number of times}

Since the universe is spatially infinite, it can be subdivided into an infinite number of regions of any given size. Thus we have an infinite number of regions and only a finite number of histories that can unfold in them. Since the regions develop independently, every possible history has a nonzero prob- 
ability and will therefore, with probability 1, occur in an infinite number of regions. (It is of course possible for an infinite universe to contain only a finite number of regions with a certain history, but the probability of that situation is strictly zero, so we will not consider it.)

Prior to Garriga and Vilenkin ([2001]), a similar argument was given by Ellis and Brundrit ([1979]), who discussed the implications of the assumptions that the universe is infinite and approximately homogeneous. They argued that there should be some regions in such a universe with histories very similar to that in our region. Our discussion here goes beyond that of Ellis and Brundrit in two respects: (i) the spatial infinity of the universe in our picture is a consequence of the theory of inflation and does not have to be independently postulated, and (ii) we argued that the number of distinct histories is finite, which allowed us to conclude that there should be regions with histories not only similar, but identical to ours (in the sense that those regions contain qualitative duplicates of every object in our region).

\section{$3 \quad$ Frequency and Probability}

The theory of inflation has surprising consequences for our intuitive understanding of frequency. On this intuitive understanding, it seems that one should be able to obtain exact frequencies by counting up the total quantities of certain objects and then doing some simple arithmetic. Thus, suppose that we are wondering about the frequency with which planets in the universe contain life. Intuitively, it may appear that the exact answer to our question could be obtained by counting up all the planets in the universe that contain life and then dividing by the total number of planets in the universe.

The theory of inflation shows that this approach is unworkable. Since the universe contains infinitely many planets and infinitely many planets that contain life, no sense can be attached to the notion of a quotient obtained by dividing the number of planets that contain life by the total number of planets. Still, there is a certain sense in which we can speak of the 'frequency' with which planets contain life. We start out by taking a finite spherical region of space. Then we can look at the ratio of the number of planets containing life to the total number of planets in that one finite region. As we increase the radius of the sphere, this ratio will converge, and the limit will not depend of the choice of the center of the sphere. The frequency with which planets in our universe contain life can then be identified with this 
limit.

Using this revised definition of frequency, it can be shown that the frequency of an event is simply equal to its quantum-mechanical probability. In other words, if quantum mechanics tells us that some given type of event occurs with probability $x$, we can infer that that type of event also occurs with frequency $x$. The quantum-mechanical probability can be defined in terms of an ensemble of qualitatively identical systems in the same initial state. The probability that a measurement of some observable will give a certain result is then simply the fraction of systems in the ensemble where that result is obtained. With eternal inflation, there is no need to introduce an imaginary ensemble: for any system, an infinite ensemble of qualitatively identical systems exists in each island universe.

We can now introduce the aspect of the theory from which the chief philosophical implications will be derived. Although there is an extremely small probability that any given region will contain a planet exactly like our ownwith exactly the same sorts of organisms, exactly the same configurations of land and ocean, and so forth - the theory of inflation nonetheless permits us to assign a probability of 1 to the proposition that there are infinitely many such planets in the universe. Moreover, the theory allows us to conclude that the universe contains infinitely many planets that diverge from ours in specific ways, with the frequency of each type of diverging planet corresponding exactly to its probability.

Thus, consider our planet as it was 300 million years ago. Given the exact state of our planet at that time, it would be possible (at least in principle) to assign quantum-mechanical probabilities to various outcomes. There was a certain probability that the planet would eventually come to contain mammals, a far smaller probability that the planet would eventually come to contain human beings, and so forth. In fact, there was a certain probability that the earth would eventually come to contain a human being exactly like you, in surroundings exactly like the ones you now inhabit, reading a philosophy paper exactly like the one you are reading right now. This last probability is extremely small - so small that we could normally afford to ignore it. But although the probability is extremely small, it is surely above zero.

The theory of inflation now allows us to conclude that, 300 million years ago, the universe contained infinitely many planets exactly like our own. These various planets then underwent various different histories, with the frequency of each history coming out precisely equal to its probability. A 
certain portion contain mammals, a smaller portion contain humans, and a still smaller portion - almost unfathomably small, but still nonzero - contain a person exactly like you.

Our own planet can therefore be seen as one element in an infinite ensemble of planets. Indeed, our planet can be seen as an element in a number of different infinite ensembles - the ensemble of all planets in the universe, the ensemble of all planets that contain intelligent life, the ensemble of all planets that are exactly like our own in every respect, and so on. In the later sections of the present paper, we argue that a number of important philosophical implications can be derived when we regard our civilization as an element of one or another of these ensembles.

\section{Inflation Contrasted}

We pause here to compare our theory with three philosophical views that may appear (at least on some superficial level) to resemble it.

Throughout this section, our chief aim is to differentiate the theory of inflation from certain philosophical views with which it might be confused. At no point will we be arguing that the theory of inflation somehow provides evidence in favor of these views. Nor will we claim that it functions as a competing theory, such that if the theory of inflation is true, these other views must be false. Rather, we claim that the philosophical views are directed primarily at questions other than the one that the theory of inflation is designed to answer. (Two of the philosophical views are concerned primarily with metaphysical questions; the third is concerned primarily with ethical questions.) By contrasting the theory of inflation with these philosophical ideas, we hope to clarify and further explain certain aspects of the theory itself.

\subsection{Modal realism}

First, we should acknowledge that the theory of modal realism, as formulated by David Lewis $([1986])$, appears to yield the very same conclusion that we have been defending thus far. Lewis is clearly committed to the view that there are infinitely many regions of any given size. Moreover, Lewis is committed to the view that every possible history is realized in at least one region. Indeed modal realism seems to yield a far stronger conclusion 
than our own, since Lewis argues that all metaphysically possible histories are realized, even those that are not physically possible. It may therefore appear that the theory of inflation is just a more complicated way of arriving at conclusions that fall naturally out of Lewis's modal realism.

But this appearance is misleading. Although the theory of inflation and modal realism seem to be making similar claims, they are in fact concerned with quite different subject matters, and they should therefore be regarded as entirely independent. Modal realism is the thesis that all possible worlds truly exist. Thus, the modal realist claims that we happen to be living in one world (the actual world) but that there are also other possible worlds and these other worlds are no less real than our own. By contrast, the theory of inflation is a thesis about the actual world. The theory makes no claims about 'other worlds' or 'parallel universes.' All of the regions posited by the theory are located in the very same spacetime that we now inhabit. Thus, when we say that one can assign a probability of 1 to the proposition that every possible history is realized in infinitely many regions, we are making a straightforward physical claim about regions of our universe. ${ }^{1}$ Most of these regions are extremely far away, but they are connected to us by ordinary spatio-temporal relations, and they all share a common causal origin.

For this reason, the theory of eternal inflation is immune to an objection that has sometimes been leveled against modal realism. The objection runs something like this: 'Since events in our own world are supposed to have no causal connection to events in other worlds, it seems that we can never really learn anything about any world other than our own. Any claim made about other possible worlds must be pure speculation, unsupported by the usual procedures of scientific inquiry.'

We do not wish to take a position either way about whether or not this is a valid objection to modal realism, but we do want to emphasize that the theory

\footnotetext{
${ }^{1}$ Thus, the theory of inflation should also be distinguished from many-world interpretation of quantum mechanics (Deutsch [1998]; DeWitt [1970]; Everett [1957]). According to this interpretation, the wave function of the universe describes a multitude of disconnected universes with all possible histories - a picture reminiscent of the one that follows from the theory of inflation. However, the reality of the other universes in the many-world theory is still a matter of controversy (see, e.g., Brown \& Davies [1993]), whereas the ensemble of regions that we discuss in this paper is unquestionably real. (We emphasize that the picture of the universe presented here is independent of the interpretation of quantum mechanics. If the many-worlds interpretation is adopted, then there is an ensemble of disconnected, eternally inflating universes, each having an infinite number of regions, where all possible histories unfold. Our picture should apply to each of the universes in the ensemble.)
} 
of inflation is not vulnerable to a parallel objection. The theory of inflation is a scientific theory, and it can therefore be supported by observational evidence. Of course, someone might argue as follows: 'All events outside the observable region are, by definition, unobservable. Therefore, we cannot gain any knowledge about events outside the observable region, and we can never know whether or not every possible history is realized in at least one region.' But this argument is without force. First of all, it isn't necessarily true that we will never be able to observe events outside the observable region. Although we are not now able to observe such events, we may be able to observe them at some future time. (Indeed, we may even be able to travel to parts of the universe that fall outside the presently observable region. ${ }^{2}$ ) More importantly, however, it seems clear that we can gain evidence about events in remote regions of the universe without ever actually observing those events. Drawing on evidence from the observable region, we can construct and test physical theories. These theories will then generate predictions about events outside the observable region, and insofar as we have reason to believe the theories, we have reason to believe the predictions they generate. In other words, even if we are never able to make observations concerning events outside the presently observable region, our knowledge of the presently observable region may permit us to make justifiable inferences concerning events in other parts of the universe.

\subsection{Actualism}

Consider now the strong form of determinism according to which nothing can possibly happen other than what actually does happen. A proponent of such a theory would say, e.g., that if we have actually decided to write this paper, we could not possibly have decided not to write the paper, indeed that our lives could not have been even slightly different from the way they actually are. Following Ayers ([1968]), we refer to this view as actualism. ${ }^{3}$

It may appear that the actualist arrives ultimately at the very same con-

\footnotetext{
${ }^{2}$ Travel to remote regions may or may not be possible, depending on the nature of the dark energy causing the accelerated expansion of the universe. If the dark energy density is constant, we will not be able to travel beyond the presently observable universe. But if the dark energy vanishes over time, then there is no limit on how far we can travel.

'In more recent work, the word 'actualism' is normally used to refer to the view that only the actual world truly exists (e.g., Adams [1981]). Note that we are here using the word in an older sense, such that it refers to the view that only actual events are possible.
} 
clusion that we have been defending thus far. After all, it seems that actualism and the theory of inflation are simply two different routes to the conclusion that everything possible is actual — with the only major difference being that actualism claims that surprisingly few things are possible whereas the theory of inflation claims that surprisingly many things are actual.

But here again, appearances are deceiving. The slogan 'Everything possible is actual' conceals an important ambiguity, and although this slogan could be appropriated with equal justice by either actualism or the theory of inflation, it would have very different meanings in these two different theoretical contexts.

The actualist asserts that there is only one possible history in any given region. By contrast, the theory of inflation does not challenge the assumption that, in any given region, there are a variety of distinct possible histories. Rather, what the theory asserts is that (with probability 1) all of these possible histories will be realized in some region of the universe. Thus, although only one of the possible histories will be actual in the region that we now inhabit, all possible histories will be actual somewhere.

In a certain sense, then, the theory of inflation is the opposite of actualism. Daniel Dennett has said that we need to 'stave off actualism' with 'elbow room' that 'prevents the possible from shrinking tightly around the actual' (Dennett [1984], p. 145, 162) The theory of inflation instead posits an infinite amount of space that permits the actual to grow to fit the possible.

\subsection{Eternal recurrence}

We turn now to a third philosophical idea that seems to resemble the theory of eternal inflation: Nietzsche's doctrine of the eternal recurrence. The doctrine is notoriously difficult to interpret, as Nietzsche's published works don't include any passages in which he presents it in his own words. All interpretations must therefore be based entirely on Nietzsche's unpublished notes and on passages from the published works in which Nietzsche presents his views through fictional stories.

In these passages, Nietzsche's fictional personae claim that everything that has happened in our lives will happen infinitely many times in the future

(e.g., $Z$ III $\S 2 ; G S \S 341)$. Nietzsche scholars disagree about how such passages should be interpreted. Some claim that Nietzsche is literally advancing a claim about the nature of the universe: namely, that every event that we now observe will recur an infinite number of times (Danto [1965]). Others say 
that the doctrine of eternal recurrence should be understood not as a literal claim about the nature of the universe but rather as a metaphor that we can use to think about our lives. On this latter view, the idea is that we ought to live our lives as though everything we did were going to recur an infinite number of times (Nehamas [1985]). Either way, it is clear that Nietzsche meant his doctrine of the eternal recurrence to have profound implications for our ordinary decisions.

Here it might be helpful to consider a more concrete example. Consider a novelist who is wondering whether to continue working on his book or just to relax for a moment and watch a sit-com on television. And now suppose the novelist comes to believe that, whichever action he chooses to perform, that action will end up being performed an infinite number of times. It seems that the novelist's decision would then acquire an enormous significance, what Nietzsche calls 'the greatest weight' ( $G S \S 341)$.

Although the theory of inflation seems at least somewhat similar to the doctrine of eternal recurrence, it would be wrong to suppose that the theory of inflation has the same implications for human life. Like the doctrine of eternal recurrence, the theory of inflation says that every action you choose to perform will be performed an infinite number of times. But unlike the doctrine of eternal recurrence, the theory of inflation also says that every possible action you choose not to perform will be performed an infinite number of times. To get a sense for the force of this claim, consider again the novelist facing a decision about how to spend his evening, this time assuming that he has come to accept the theory of inflation. The novelist will then conclude that there are infinitely many people exactly like him and that each member of this infinite ensemble faces a choice between working and watching television. However, he will not feel that these other people stand to him in any relation of causal dependence. ${ }^{4}$ Nor will he believe that their choices must necessarily be identical to his own. On the contrary, he

\footnotetext{
${ }^{4}$ Here our novelist appears to be faced with a complex problem in decision theory. If he chooses to work on his novel, he will be maximizing the expected frequency with which his counterparts throughout the universe chose to work on their respective novels. (After all, it is highly probable that the majority of his counterparts will end up choosing the same option that he himself chooses.) But since he cannot actually have any causal impact on these counterparts, we will assume that it would be a mistake for him to try to maximize the expected frequency with which they perform a particular action. In other words, we will presuppose that he ought to act in accordance with some version of causal decision theory.
} 
will reach precisely the opposite conclusion: that no matter which option he chooses to select, an infinite number of people exactly like him will end up selecting some other option. Thus, he will conclude that, even if he chooses to relax and watch television, an infinite number of people exactly like him will choose to keep working on the novel.

\section{Ethical Implications}

Since the theory of inflation leads in this way to the opposite conclusion from the doctrine of eternal recurrence, one might think that the theory of inflation should have the opposite effect on the way people think about their lives. Just as the doctrine of eternal recurrence makes every decision seem extremely weighty or important, one might think that the theory of inflation makes every decision seem insignificant or inconsequential. A defender of such a view could say: 'We already know (with probability 1) that infinitely many good events will occur and that infinitely many bad events will occur. We know, for example, that infinitely many people exactly like our novelist will finish their work and that infinitely many will leave their work unfinished. Nothing that anyone does can ever change this. So why should it be a matter of any real concern whether some given person happens to choose one option or the other?'

To evaluate this argument, we need to distinguish among a number of different ways in which a person might have a deep concern with her own decisions. We can then ask, for each of these types of concern, what impact the theory of inflation ought to have.

First, let us consider irreducibly de se concerns - i.e., concerns that relate in some essential way to one's own self (Castañeda [1966]; Lewis [1979]). To take a simple example, imagine a person who wants to go jogging. Presumably, her aim is not that all people of some general type go jogging. Rather, her aim is that she herself go jogging. To the extent that a person's concerns have this de se character, they should be relatively unaffected by knowledge of the theory of inflation. After all, suppose the person knows full well that there are infinitely many people exactly like her, and suppose she knows that, no matter what she does, infinitely many of these people will go jogging and infinitely many will not go jogging. This knowledge may have little

or no bearing on her real concern. Her concern is not with what happens to all of those other people but with what happens to her. She is concerned 
about whether or not she ends up going jogging, and the fact that there are infinitely many people exactly like her seems not to affect the issue in any way.

Similar remarks apply to those who are concerned with particular objects, events or people. Take the father who feels a special concern for his own daughter. Even if he discovers that remote regions of the universe contain other people who resemble his daughter in every possible respect, he might find that he cares far more about his own daughter than he does about any of these other people (Frankfurt [1999]). Suppose, e.g., that such a man sees his daughter crying and runs to comfort her. If he accepts the theory of inflation, he can conclude that the universe contains an infinite number of events exactly like the one he is now witnessing - an infinite number of girls exactly like his daughter, all feeling upset in exactly the same way for exactly the same reason. However, this conclusion will not lead him to regard his own action as any less consequential. He will not feel frustrated to learn that he is helping only one member of an infinite population. Rather, he will feel that his own daughter has some special importance - an importance that no other person can share - and that he is therefore accomplishing something important by making sure that she receives adequate comfort.

But now suppose we turn to a person who is concerned with the total quantity of something in the world. Such a person might donate money to the Audubon Society in the hope of increasing the total quantity of goldfinches. Or, in a more philosophical moment, the person might think that morality is a matter of increasing the total quantity of happiness in the universe. Here there really does seem to be a problem. If there are infinitely many goldfinches in the world, it seems that one cannot increase their total quantity by donating to the Audubon Society. Similarly, if there is already an infinite quantity of happiness, one cannot increase that quantity by engaging in altruistic activities. (Of course, one can engage in activities that cause some people to be happier and don't cause any people to be less happybut this result is not correctly described as involving a net increase in any total quantity.) To determine whether or not this sort of concern should be affected by knowledge of inflation, one has to ask oneself whether it is truly the total quantity that matters. Is it necessary that one actually increase the total quantity of happiness? Or would it be sufficient merely to perform an action that added some happiness to the world without subtracting any away? Or would it perhaps be sufficient to increase the total quantity of happiness around here without having any effect at all on the total quantity 
in the world as a whole?

Note that the problems that arise here are very different from those facing the modal realist. The modal realist cannot coherently say that we ought to try, through our actions, to make reality as a whole better than it would otherwise have been. Of course, the modal realist can still use sentences like: 'Things would be better if you gave that money to the Audubon Society than if you gave it to the Nazi Party.' But such a sentence means only that the possible world in which you give to the Audubon Society is better than the one in which you give to the Nazi Party. No real sense can be attached to the notion that reality as a whole -including all of the possible worlds would be better if you did one thing rather than another. Faced with this difficulty, Lewis ([1986] p. 128) suggests that we give up on the whole idea of a universalistic ethics. Instead, he argues that we ought to show a particular concern for the people who occupy our own world.

Eternal inflation is not nearly so radical in its implications. A believer in eternal inflation can still hold on to the view that the universe would be better if you did one thing rather than another. It's just that this notion of the universe's being 'better' cannot be understood in terms of maximizing the total quantity of happiness. To the extent that one wants to hold on to the underlying spirit of utilitarianism, one will have to make certain technical modifications in its 'total happiness principle.' Such modifications have been proposed by Vallentyne and Kagan ([1997]) among others (see Bostrom [2004]) . We will not be getting into the details here, but one key implication is that an agent is doing the right thing if he or she increases the total quantity of happiness of the people in some finite set and has no effect on anyone else.

Finally, let us consider cases in which a person is specifically concerned with uniqueness. An art collector may value a particular painting on the grounds that, in the whole world, there has never been anything quite like it. A scientist may derive a special kind of pride from thoughts like 'I am the only person ever to have developed this key insight.' An environmentalist may ascribe a special importance to a specific herd of animals on the grounds that they are the only remaining specimens of their species. Here again, the theory of inflation may indicate that something has gone wrong. When claims of uniqueness are taken in the most literal sense, the theory of inflation can show that they are false. Thus, the art collector is wrong to think that there are literally no paintings in the entire world exactly like the one she now possesses. The truth is that there are infinitely many paintings exactly 
like hers; it's just that they are so far away that she will never be able to observe them. The important question, then, is whether it really matters that a particular object or event be literally unique. Does it really matter, for example, that the painting be literally the only one of its kind in the entire universe? Or is it sufficient that the painting be the only one of its kind within a $10^{100}$ parsec radius?

This sort of question becomes especially pressing when applied to the concern we feel about the continuing existence of our own civilization. The theory of inflation tells us that the universe contains an infinite number of civilizations exactly like ours. Thus, even if our own civilization is entirely destroyed over the course of the next century, the theory tells us that an infinite number of other civilizations exactly like ours will continue to exist. Does the theory therefore give us a reason to feel less concerned about nuclear wars, asteroid collisions and other events that might destroy our civilization? Here again, the answer will depend on why exactly we were concerned about the possibility of this destruction in the first place. If we were concerned because we valued particular people or particular institutions that now inhabit the earth, then the theory should have no effect on our feelings. But if we were concerned because we felt that our civilization was somehow unique-so that if our civilization were destroyed, the universe would no longer contain anything even remotely like the presently-existing human race - then the theory tells us that our concern was based on a false assumption.

Presumably, the concern that we actually feel is based on a complex combination of different beliefs, desires and emotions. Some of these should be affected by the theory of inflation; others should not. It therefore remains to be seen whether the theory should have any substantial impact on our overall attitude toward the continuing existence of our civilization.

\section{Universal Doomsday}

As discussed above, the theory of inflation implies that we are part of an infinitely large 'island universe' that contains an infinite number of civilizations. According to the anthropic principle (codified, for example, as the 'self-sampling assumption'; Bostrom [2002]) we should reason as if we were randomly selected from all the individuals in all those civilizations. Thus our expectation of finding ourselves in any particular circumstances is proportional to the number of observers in those circumstances. We now want 
to ask whether it is possible to use information about our own circumstances to make inferences about the average lifetimes of civilizations in our universe.

First, it is clear that there is some nonzero probability for a civilization to survive early threats to its existence (nuclear war, asteroid impact, etc.). Such a civilization might go on to spread across its galaxy. It could endure for millions of years and contain a huge number of individuals. We will refer to such civilizations as long-lived. On the other hand, some civilizations will succumb to existential threats and so be short-lived. What will be the fraction of each?

Unless the fraction of long-lived civilizations is tiny, nearly all individuals will belong to them, and furthermore will live late in their civilizations when most of the individuals live. That, however, is not the circumstance in which we find ourselves. Instead, we find that we live either in a short-lived civilization or very early in a long-lived one. While we do not have a clear idea of how long to expect civilizations to last, when we take into account our circumstances, we should clearly update our ideas in favor of a much larger chance for civilizations to be short-lived (Carter unpublished; Leslie [1996] p. 231; see also Olum [2004]).

Let $f(S)$ be the frequency (as defined in 93 ) with which civilizations are short-lived, and $f(L)=1-f(S)$ the frequency with which civilizations are long-lived. We don't know these frequencies, so let $f_{\text {prior }}$ denote our best understanding of the frequencies before we take into account our circumstances. Now suppose you learn that you have birth rank $N$, i.e., you are the $N$ th human to be born. You should update your frequencies via Bayes's Rule, to get

$$
f_{N}(S)=\frac{P(N \mid S) f_{\text {prior }}(S)}{P(N \mid S) f_{\text {prior }}(S)+P(N \mid L) f_{\text {prior }}(L)},
$$

where $P(N \mid a)$ is the chance that you would have birth rank $N$ given that we are in a long- or short-lived civilization, and $a$ is $L$ or $S$ accordingly. You could be any individual in the civilization, so

$$
P(N \mid a)= \begin{cases}1 / N_{a} & N \leq N_{a} \\ 0 & N>N_{a}\end{cases}
$$

where $N_{a}$ is the number of individuals in each civilization. Since $N_{L} \gg N_{S}$, $P(N \mid L) \ll P(N \mid S)$, and thus $f_{N}(S)$ is nearly 1 unless $f_{\text {prior }}(S)$ is extremely small. Unless we previously thought that long-lived civilizations were much more likely, we should now think that almost all civilizations will be shortlived - a sort of 'universal doomsday'. 
The 'universal doomsday' argument that we advance here should be carefully distinguished from the classic doomsday argument (Carter unpublished; Gott [1993]; Leslie [1989], [1996]; Nielsen [1989]). The classic doomsday argument was an attempt to show that our present circumstances give us some reason to believe that our own particular civilization will soon come to an end. The argument advanced here is quite different. We make no specific claims regarding the longevity of any particular civilization. Rather, we say that our present circumstances give us reason to reach a general conclusion about our universe: namely, that long-lived civilizations are extremely infrequent in our universe as a whole.

Moreover, as we now proceed to argue, the theory of inflation gives us reason to reject the particular doomsday argument, accepting only the universal doomsday argument. Thus, the doomsday argument has nothing to say specifically about our own civilization as distinct from others. Instead it tells us about the general longevity of civilizations sufficiently similar to ours to be included in the same reference class - although, of course, what we learn about civilizations in general, we should also apply to ourselves.

\subsection{Application to our civilization in particular}

Traditionally, the doomsday argument has been applied to the future of our own particular civilization. We let $P_{\text {prior }}(a)$, with $a=S$ or $L$, be our subjective probabilities that our own civilization will be short-lived or long-lived, before we take into account our birth rank. We then update our probabilities as

$$
P(S \mid N)=\frac{P(N \mid S) P_{\text {prior }}(S)}{P(N \mid S) P_{\text {prior }}(S)+P(N \mid L) P_{\text {prior }}(L)}
$$

Thus unless $P_{\text {prior }}(S)$ is infinitesimal, we will find that $P(S \mid N)$ is nearly 1 , just as above.

That analysis, however, neglects the possibility that we could have been in any other civilization. There is some controversy about which individuals should be included in the reference class among which we should expect to be typical, but it should be clear that we must at least include all observers subjectively indistinguishable from ourselves (Bostrom [2002]). However, the theory of inflation implies that there are infinitely many such observers, belonging to civilizations with every possible lifespan.

Now we should reason as though we were chosen randomly among humans in the various human civilizations in our universe, where again the random 
choice is to be understood as the limit of random choices in successively larger finite regions, as in 93 . Then, before we make the probability shift above, we should realize that the chance that our civilization is large is not just the frequency of civilizations that are large, but must be corrected for the increased chance to be in a long-lived civilization because it has more individuals. Taking that into account, we find that the probability for our civilization to be small, before taking into account birth rank, is

$$
P_{\text {prior }}(S)=\frac{N_{S} f(S)}{N_{S} f(S)+N_{L} f(L)}
$$

and similarly for $P_{\text {prior }}(L)$. To take our birth rank into account, we use (4) in (3) to get

$$
P(S \mid N)=f(S) .
$$

This effect exactly cancels out the impact of the particular doomsday argument, leaving us with the conclusion that our chances that we are now in a long-lived or a short-lived civilization are just proportional to the prevalence of such civilizations (Bostrom [2002]; Dieks [1992]; Olum [2002]). Thus, if the theory of inflation is correct, the doomsday argument has nothing to say about the longevity of our specific civilization, but only about the general longevity of civilizations sufficiently similar to ours to be included in the same reference class.

At this point, one might object that similar considerations could be used to defeat the universal doomsday argument. Thus, one might suggest that we are typical not merely among all those individuals in our universe, but rather among all those individuals who might exist according to alternative theories of the universe, if we don't know which theory is correct. If the universe developed in some probabilistic way before the beginning of inflation, so that early chance events affected all regions together, then one can consider also the possible observers who might exist as a result of all different early developments. Including all such possible observers in the reference class is equivalent to accepting the self-indication assumption (SIA) (Bostrom [2002]), first introduced by Dieks ([1992]), which states that the chance that you would exist at all is greater in a universe which contains more observers. If one accepts SIA, then a universe with long-lived civilizations is more likely because of the greater number of individuals that it contains, and that effect cancels all forms of doomsday argument. SIA is controversial, however, most notably because of the 'presumptuous philosopher' example (Bostrom 
[2002]). We will not address that controversy here ${ }^{5}$, but for the purposes of the present paper will consider the situation where one does not include SIA.

\subsection{Universal vs. particular dooms}

Some effects which might shorten the life expectancy of our civilization apply only to ours specifically, while others shorten the general life expectancy of all civilizations. For example, suppose that we are concerned with the earth being hit by an asteroid. The chance of such a collision, in the next century say, is a function of the number of asteroids in the solar system and the chance that any given asteroid is on a course which will hit the earth during that period.

Now a specific asteroid which happens to be on a collision course with us is a 'particular doom' that affects only us. The fact that the asteroid has, by chance, the doomsday orbit says nothing about other asteroids in other solar systems like ours. The particular orbit of the asteroid is unrelated to the distribution of civilizations that will or will not be destroyed by asteroids. Given the theory of inflation, there is thus no reason to believe that such orbits are more likely than one would first think.

On the other hand, the total number of asteroids could well be determined by some universal process of solar system formation and most solar systems like ours would have similar numbers of asteroids. Therefore if the (incompletely known) process that produces asteroid belts turns out to produce an especially large number of asteroids, the lifetimes of all civilizations would be on average shortened. Large numbers of asteroids are a 'universal doom' that (statistically) affects all civilizations, and thus the doomsday argument makes them more likely.

\subsection{Practical applications}

The doomsday argument has practical applications. If you care about the future of the human race you might want to reduce the probability of possible causes of extinction that you might have some control over. The degree to which you should be concerned with such possible causes depends on how likely they are, and if you accept the doomsday argument you should think the possible dooms more likely than you would otherwise have thought.

\footnotetext{
${ }^{5}$ For recent discussions of arguments for and against SIA, see Olum ([2002]) and Bostrom and Cirkovic ([2003]).
} 
The argument presented here should change your calculation. You should not think a process is more likely if it affects us alone, but you should think it more likely if it affects civilizations everywhere in the universe. To continue the above example, you should be more concerned that a large number of asteroids have not yet been detected than about the particular orbit of each one. You should not worry especially about the chance that some specific nearby star will become a supernova, but more about the chance that supernovas are more deadly to nearby life than we believe. Many other examples are possible.

\section{Concluding Remarks}

Since at least the time of Copernicus, physicists have been casting doubt on the naïve view that our planet plays some unique and special role in the universe. First it became clear that our planet was not the center of the cosmos - that the planet Earth was just one of the planets in our solar system. Then we gradually accumulated evidence for the view that our solar system was itself just one of the many such systems in the universe. These theoretical advances contributed to a growing sense that our civilization plays no special role in the cosmic drama, that it is just one tiny speck in a vast universe. Thus, a series of scientific discoveries led to a series of philosophical problems - problems about the significance of human life, about our role in the divine plan, and so forth.

But although scientific discoveries have done a great deal to threaten our naïve worldview, they did appear to leave us with one way of holding on to our intuitive sense that there was something special and unique about the planet earth. We knew that our planet was just one of the many planets in the universe, but we could nonetheless hold on to the idea that it was the only planet that had certain distinctive properties - probably the only planet with anything remotely like a human being, certainly the only one with all the art forms, cultural traditions and political institutions that we most associate with life on earth. The theory of inflation now shows us that even this last claim to uniqueness was, in fact, illusory. Even we ourselves are not unique; in that sense, as Alan Guth has said, the theory shows that we do not even have 'a unique copyright on our own identities' (Quoted in Martin [2001]). This new theoretical advance casts up a set of new philosophical questions; we have tried to begin the exploration of those questions here. 


\section{Acknowledgments}

We are grateful to Nick Bostrom, Brandon Carter, Daniel Dennett, Jaume Garriga and two anonymous referees for useful discussions and comments. The work of K.D.O. and A.V. was supported in part by the National Science Foundation.

Philosophy Department, Princeton University Princeton, NJ 08544-1006

jknobe@Princeton.edu

Department of Physics and Astronomy, Tufts University

Medford, MA 02155

kdo@cosmos.phy.tufts.edu

Department of Physics and Astronomy, Tufts University

Medford, MA 02155

vilenkin@cosmos.phy.tufts.edu 


\section{References}

Adams, R. [1981]: 'Actualism and Thisness', Synthese, 49, pp. 3-41.

Ayers, M. [1968]: The Refutation of Determinism, London: Methuen.

Bostrom, N. [2002]: Anthropic Bias: Observation Selection Effects, New York: Routledge.

Bostrom, N. [2004]: 'Infinite Ethics',

http://www.nickbostrom.com/ethics/infinite.pdf

Bostrom, N. and M. Cirkovic [2003]: 'The Doomsday Argument and the Self-Sampling Assumption: Reply to Olum', Philosophical Quarterly, 53, pp. 83-91.

Brown, J. and P. C. W. Davies (eds.) [1993]: The Ghost in the Atom (Cambridge: Cambridge University Press).

Castañeda, H.-N. [1966]: 'He: A Study in the Logic of Self-Consciousness', Ratio, 8, pp. 130-57.

Danto, A. [1965]: Nietzsche as Philosopher, New York: Macmillan.

Dennett, D. [1984]: Elbow Room: The Varieties of Free Will Worth Wanting, Cambridge, Mass.: MIT Press.

Deutsch, D. [1998]: The Fabric of Reality, London: Penguin Books.

DeWitt, B. [1970]: 'Quantum Mechanics and Reality', Physics Today, 23, pp. 30-40.

Dieks, D. [1992]: 'Doomsday — or: The Dangers of Statistics', Philosophical Quarterly, 42, pp. 78-84.

Ellis, G. F. R. and G. B. Brundrit [1979]: 'Life in the Infinite Universe', Quarterly Journal of the Royal Astronomical Society, 20, pp. 37-41.

Everett, H. [1957]: 'Relative-State Formulation of Quantum Mechanics', Reviews of Modern Physics, 29, pp. 454-62.

Frankfurt, H. [1999]: 'On Caring', In his Necessity, Volition, and Love (Cambridge: Cambridge University Press).

Garriga, J. and A. Vilenkin [2001]: 'Many worlds in one', Physical Review $D, \mathbf{6 4}, 043511$.

Gell-Mann, M. and J. B. Hartle [1993]: 'Classical Equations For Quantum Systems,' Physical Review D, 47, pp. 3345-82

Gott, J. R. III [1993]: 'Implications of the Copernican Principle for our Future Prospects', Nature, 363, pp. 315-9. 
Guth, A. [1981]: 'The Inflationary Universe: A Possible Solution to the Horizon and Flatness Problems', Physical Review D, 23, pp. 347-56.

Guth, A. [2000]: 'Inflation and Eternal Inflation', Physics Reports, 333, pp. $555-74$.

Leslie, J. [1989]: 'Risking the World's End', Bulletin of the Canadian Nuclear Society, 10, pp. 10-5.

Leslie, J. [1996]: The end of the world, London: Routledge.

Lewis, D. [1979]: 'Attitudes De Dicto and De Se', The Philosophical Review, 88, pp. 513-43.

Lewis, D. [1986]: On the plurality of worlds, Oxford: Blackwell.

Linde, A. [1986]: 'Eternally Existing Self-Reproducing Chaotic Inflationary Universe', Physics Letters, B175, pp. 395-400.

Martin, M. [2001]: 'Other Worlds May Surround Us, Physicists Claim', United Press International, http://www.sciencenewsweek.com/articles/worlds.htm.

Nehamas, A. [1985]: Nietzsche: Life as literature, Cambridge, Mass.: Harvard University Press.

Nielsen, H. B. [1989]: 'Random Dynamics and Relations Between the Number of Fermion Generations and the Fine Structure Constants', Acta Physica Polonica B, 20, pp. 427-68.

Nietzsche, F. [1969]: Thus spoke Zarathustra [Cited as Z.] Trans. R. J. Hollingdale (London: Penguin Books).

Nietzsche, F. [1974]: The Gay Science [Cited as GS.] Trans. W. Kaufmann. New York: Random House.

Nietzsche, F. [1968]. The Will to Power [Cited as WP.] Trans. W. Kaufmann and R. J. Hollingdale, New York: Random House.

Olum, K. D. [2002]: 'The doomsday argument and the number of possible observers', Philosophical Quarterly, 52, pp. 164-84.

Olum, K. D. [2004]: 'Conflict between anthropic reasoning and observation', Analysis, 64, pp. 1-8.

Richards, T. [1975]: 'The Worlds of David Lewis', Australasian Journal of Philosophy, 53, pp. 105-18.

Smolin, L. [2001]: Talk given at the conference Anthropic arguments in fundamental physics, Cambridge.

Vallentyne, P. and S. Kagan [1997]: 'Infinite Value and Finitely Additive 
Value Theory', The Journal of Philosophy, 94, pp. 5-26.

Vilenkin, A. [1983]: 'The birth of inflationary universes', Physical Review D27, pp. 2848-55.

Vilenkin, A. [1998]: 'Unambiguous probabilities in an eternally inflating universe', Physical Review Letters 81, pp. 5501-4. 